\title{
Diacronie
}

Studi di Storia Contemporanea

$N^{\circ} 17,1 \mid 2014$

Periferie. Cultura, economia, politica

\section{Matteo Guglielmo, Il Corno d'Africa. Eritrea, Etiopia, Somalia}

\section{Michele Pandolfo}

\section{Q OpenEdition}

\section{Edizione digitale}

URL: http://journals.openedition.org/diacronie/1146

DOI: 10.4000/diacronie.1146

ISSN: 2038-0925

Editore

Association culturelle Diacronie

Notizia bibliografica digitale

Michele Pandolfo, «Matteo Guglielmo, Il Corno d'Africa. Eritrea, Etiopia, Somalia », Diacronie [Online], N

17, 1 | 2014, documento 15, Messo online il 01 mars 2014, consultato il 24 septembre 2020. URL :

http://journals.openedition.org/diacronie/1146 ; DOI : https://doi.org/10.4000/diacronie.1146 


\section{Diacronie}

N. 17 | 1|2014 Periferie. Cultura, economia, politica

15/

\section{RECENSIONE:}

\section{Matteo GUGLIELMO, Il Corno d'Africa. Eritrea, Etiopia, Somalia, Bologna, Il Mulino, 2013, 190 pp.}

a cura di Michele PANDOLFO *

Il nuovo lavoro di Matteo Guglielmo' si intitola Il Corno d'Africa. Eritrea, Etiopia, Somalia pubblicato dalla casa editrice Il Mulino nella collana Universale Paperbacks nei primi mesi del 2013.

Questo testo cerca di analizzare una regione del mondo che è sempre stata al centro degli interessi politici ed economici sia delle grandi potenze europee - come Gran Bretagna e Francia -, sia degli Stati Uniti d'America e dell'Unione Sovietica durante gli anni della Guerra Fredda. La complessità storica, geografica e politica di questa regione africana porta Matteo Guglielmo a cercare un filo conduttore lungo il quale costruire una narrazione approfondita del Corno d'Africa. Questa prima difficoltà incontrata nella ricerca ben si evince sin dalle prime parole dell'autore che a tal proposito afferma:

\footnotetext{
Su questo punto ho trovato utile considerare proprio il "conflitto" come il tratto distintivo che attraversa - a vari gradi e livelli - tutto il Corno d'Africa, e l'approccio cronologico il miglior metodo di rappresentazione, in quanto la sequenzialità degli eventi può forse rendere più visibili quelle che sono le ciclicità storiche che continuano a segnare quest'area. Ciò che si può affermare con certezza,
}

\footnotetext{
${ }^{1}$ Matteo Guglielmo è dottore di ricerca in Studi africani e membro del Centro studi sull'Africa contemporanea (CeSAC) presso l'Università Orientale di Napoli. Tra le sue pubblicazioni ricordo: GUGLIELMO, Matteo, «La crisi di confine tra Eritrea ed Etiopia apre un altro fronte in Somalia», in Afriche e Orienti, 3, 4/2006, pp. 119-123; ID., Somalia: le ragioni storiche del conflitto, Pavia, Altravista, 2008; ID., "Dinamiche politiche e regimi di conflitto nella Mogadiscio contemporanea» in Storia Urbana, 126-127, 1/2010, pp. 17-36; ID., Somalia: conflitti interni e destabilizzazione regionale, Milano, ISPI, 2011; ID., Unravelling the Islamist Insurgency in Somalia: The Case of Harakat al-Shabaab Mujahideen, in SHARAMO, Roba, MESFIN, Berouk (ed. by), Regional Security in the Post-Cold War Horn of Africa, Pretoria, Institute for Security Studies, 2011, pp. 119-146; GUGLIELMO, Matteo, La Somalia dal collasso alla frammentazione: la posta in gioco a Londra, Milano, ISPI Analysis, 2012.
} 
trovando riscontro sia nel passato sia nel presente, è che il Corno d'Africa è in perenne conflitto ${ }^{2}$.

Dopo aver trovato il termine che sarà il perno attorno al quale far emergere la ricerca, un'altra questione d'affrontare è quella della posizione geografica di una così complessa regione. L'autore, prima di tutto, si chiede quali siano i confini naturali del Corno d'Africa e quali Stati si possano inglobare politicamente in tale definizione:

[...] la sua particolare posizione geografica tra Africa e Medio oriente pone l'intero complesso regionale al centro di importanti interessi globali, dalla lotta al terrorismo al contrasto della pirateria marittima. Le numerose crisi ivi presenti hanno radici profonde e peculiari, tanto che non sarebbe del tutto impreciso definire il Corno d'Africa quasi un'invenzione dettata più dalla necessità di inquadrare un'area di congiunzione tra il continente africano e il Medio Oriente, che dalla sua effettiva uniformità sociopolitica. Per convenzione, da un punto di vista geografico, il Corno d'Africa è spesso identificato come l'insieme dei paesi membri dell'IGAD (Inter-Governmental Authority on Development), l'organizzazione regionale che comprende Eritrea, Etiopia, Somalia, Gibuti, Uganda, Kenya, Sudan e Sud Sudan. Nel testo si è preferito tuttavia concentrare l'oggetto di studio ai primi tre stati, che presi sia singolarmente sia nel loro insieme possono meglio rappresentare le caratteristiche politiche e conflittuali della regione 3 .

La linea cronologica scelta da Matteo Guglielmo parte dalla storia antica del Corno d'Africa che ha rappresentato nel corso dei secoli un insieme di migrazioni, spostamenti, crocevia, incontri culturali tra diverse popolazioni e scambi economici tra l'Africa, la penisola arabica e l'Asia. L'espansione coloniale europea ha determinato, anche in questa parte dell'Africa, la divisione in zone d'influenza diplomatica ed economica che in seguito si sono trasformate in vere e proprie aree di dominio politico e militare. I paesi europei che hanno sviluppato i loro interessi in questa regione sono stati la Gran Bretagna, la Francia e anche l'Italia4. Come ben ricorda Matteo Guglielmo,

\footnotetext{
${ }^{2}$ GUGLIELMO, Matteo, Il Corno d'Africa. Eritrea, Etiopia, Somalia, Bologna, Il Mulino, 2013, p. 9.

3 Ibidem, pp. 9-10.

4 Per una bibliografia ragionata di studi italiani sul colonialismo rimando brevemente ai seguenti titoli: ROCHAT, Giorgio, Il colonialismo italiano, Torino, Loescher, 1974; DEL BOCA, Angelo, Gli italiani in Africa Orientale, 4 voll., Roma-Bari, Laterza 1976-1984; DEL BOCA, Angelo, L'Africa nella coscienza degli italiani: miti, memorie, errori, sconfitte, Roma-Bari, Laterza, 1992; GOGLIA, Luigi, GRASSI, Fabio, Il colonialismo italiano da Adua all'impero, Bari, Laterza, 1993; CALCHI NOVATI, Giampaolo, Il Corno d'Africa nella storia e nella
} 
infatti, la debole Italia postunitaria trova in questa regione d'Africa una concreta possibilità di realizzare le proprie mire espansionistiche e coloniali, inserendosi con circospezione e talvolta ambiguità tra le rivalità diplomatiche delle altre potenze europee. L’interesse italiano più antico è quello per la fascia costiera affacciata sul Mar Rosso chiamata Eritrea. In seguito l'Italia occupa le regioni costiere somale meridionali, mentre la Gran Bretagna quelle della Somalia settentrionale, mentre la Francia si impossessa di Gibuti, punto strategico di passaggio per le rotte commerciali nel golfo di Aden. Gli italiani tentano poi per decenni di espandere inutilmente la propria influenza sul grande impero etiopico.

Il testo di Matteo Guglielmo si divide in tre capitoli che, dopo aver riassunto brevemente la storia antica del Corno d'Africa, intraprendono un'analisi più approfondita della regione a partire dalla fine della Seconda guerra mondiale e si soffermano sui movimenti politici interni sorti nelle ex colonie europee per il raggiungimento delle singole indipendenze nazionali. L'attenzione dello storico si concentra ciclicamente verso tutti e tre i maggiori paesi oggetto d'analisi - l'Eritrea, l'Etiopia e la Somalia - attraversandone le varie fasi storiche che comprendono anche i conflitti militari scoppiati nella regione e le alleanze con Stati Uniti d'America e Unione Sovietica. In questa maniera l'autore analizza tutti i passaggi storici più importanti: la delicata fase della decolonizzazione, la federazione dell'Eritrea all'impero etiopico, il periodo dell'Amministrazione fiduciaria italiana della Somalia, la nascita della repubblica somala e l'avvento della dittatura di Siad Barre, la caduta del Negus Hailé Selassié in Etiopia e l'avvento al potere della giunta militare del Derg dalla quale emergerà la figura politica di Menghistu, le drammatiche guerre tra Somalia ed Etiopia per la regione frontaliera dell'Ogaden e l'inizio della guerriglia eritrea contro il dominio etiopico. Tutti questi processi storici vengono analizzati da Matteo Guglielmo sempre all'interno della grande cornice geopolitica globale rappresentata dallo scontro tra gli Stati Uniti d'America e l'Unione Sovietica. Entrambi questi paesi cercano continuamente nel corso dei decenni di spartirsi nuove zone d'influenza nella cartina geografica mondiale, ma questa perenne ingerenza risulta particolarmente pesante nel Corno d'Africa che viene coinvolto e poi abbandonato a se stesso sull'onda di scelte politiche che vengono prese senza tener conto delle vere problematiche e delle esigenze della regione.

politica: Etiopia, Somalia e Eritrea fra nazionalismi, sottosviluppo e guerra, Torino, SEI, 1994; LABANCA, Nicola, Oltremare: storia dell'espansione coloniale italiana, Bologna, Il Mulino, 2002; DEL BOCA, Angelo, Italiani brava gente? Un mito duro a morire, Vicenza, Neri Pozza, 2005; STEFANI, Giulietta, Colonia per maschi. Italiani in Africa Orientale: una storia di genere, Verona, Ombre corte, 2007; CALCHI NOVATI, Giampaolo, L'Africa d'Italia. Una storia coloniale e postcoloniale, Roma, Carocci, 2011. 
Il traumatico momento di svolta per i principali paesi della regione è rappresentato proprio dalla caduta di questo sistema di dominio che è cessato con lo scioglimento dell'Unione Sovietica e la conseguente fine della Guerra fredda. Infatti proprio agli inizi degli anni Novanta anche i due principali regimi del Corno d'Africa, quello etiopico e quello somalo, sono crollati portando l'intera regione a livelli di instabilità mai raggiunti prima. Al crollo della dittatura in Etiopia ha fatto seguito la guerra civile con l'Eritrea, che diventerà indipendente nel 1993, e un delicato processo di democratizzazione all'intero dell'ex impero etiopico. In Somalia, invece, la situazione generale si è evoluta in maniera notevolmente più complessa. Allo scoppio della guerra civile nel gennaio del 1991 è seguita la fuga del dittatore Siad Barre e l'inizio di una guerra clanica che ha distrutto qualsiasi forma statuale all'interno dei vecchi confini politici della Somalia. La delicata fase d'intervento internazionale negli anni 1992-1993 con la missione Restore Hope delle Nazioni Unite avviene parallelamente ai vari tentativi falliti di riconciliazione nazionale rappresentati da alcune conferenze di pace.

Dopo la fase del disimpegno internazionale dal Corno d'Africa, e in particolare nel conflitto somalo, seguono gli attentati dell'11 settembre 2001: questi cambiano completamente l'agenda mondiale, che adotta come nuove ragioni del conflitto il pericolo del terrorismo internazionale soprattutto di matrice islamica e la lotta per contrastarlo. In questo nuovo quadro generale, che pure coinvolge la regione del Corno d'Africa, Matteo Guglielmo individua i traffici delle grandi organizzazioni terroristiche, le rotte problematiche della pirateria internazionale e l'ingerenza dell'Etiopia nel caos somalo con l'obiettivo dello scioglimento delle Corti Islamiche che detenevano il controllo di molte città, tra cui l'ex capitale Mogadiscio. Ed è proprio la Somalia a essere tornata al centro dell'instabilità dell'intera regione orientale africana, come afferma Guglielmo:

Il conflitto somalo è oggi il fulcro attorno al quale sembra reggersi "l'equilibrio instabile" del Corno d'Africa, che dopo vent'anni dal suo inizio può essere considerato ormai strutturale. Mogadiscio si trova direttamente esposta a tutte le grandi tendenze politiche, sociali ed economiche globali. Per capirne il sistema, quindi, è necessario riportare alla luce quei processi politici sotterranei che i molti anni di instabilità hanno contribuito a mascherare. E' proprio attraverso un'analisi degli attori, e del loro agire in più contesti, che è forse possibile dare un senso ai conflitti complessi emersi in questa zona di confine tra Africa e Medio oriente martoriata da faide e scontri armati apparentemente senza fine 5 .

5 GUGLIELMO, Matteo, Il Corno d'Africa. Eritrea, Etiopia, Somalia, cit., p. 11. 
Il nuovo testo di Matteo Guglielmo, che risulta di facile lettura, presenta caratteristiche originali che interessano non solo il lettore più attento e specialista, ma anche un pubblico più vasto. Inoltre il giovane studioso dedica in maniera esaustiva la parte finale della sua ricerca ai passaggi storici degli anni Novanta e Duemila, con particolare attenzione sia all'intervento internazionale in Somalia durante le fasi iniziali della guerra civile, sia all'arrivo nel contesto somalo di alcuni gruppi terroristici di matrice islamica. Il volume è fornito di un buon apparato cartografico e di quattro schede esplicative che riassumono alcuni temi di difficile comprensione: Il ruolo dei clan in Somalia, Le etnie dell'Etiopia, L'Eritrea e la geopolitica del confine e La riforma dell'Unione africana e il ruolo di AMISOM. Infine la bibliografia è ben strutturata e ricostruisce i maggiori studi italiani e internazionali riguardanti la complessa regione del Corno d'Africa. 


\section{* L'autore}

Michele Pandolfo ha conseguito la Laurea Specialistica in Filologia Moderna; è oggi Dottorando in Storia, Culture e Strutture delle Aree di Frontiera presso l'Università degli Studi di Udine, in cotutela con l’Université Paul Valery di Montpellier.

URL: < http://www.studistorici.com/progett/autori/\#Pandolfo >

\section{Per citare questo articolo:}

PANDOLFO, Michele, «Recensione: Matteo GUGLIELMO, II Corno d'Africa. Eritrea, Etiopia, Somalia, Bologna, ॥I Mulino, 2013, 190 pp», Diacronie. Studi di Storia Contemporanea : Periferie. Cultura, economia, politica, 29/3/2014, URL: < http://www.studistorici.com/2014/3/29/pandolfo_numero_17/ >

Diacronie Studi di Storia Contemporanea $\beta$ www.diacronie.it

Risorsa digitale indipendente a carattere storiografico. Uscita trimestrale.

redazione.diacronie@hotmail.it

Comitato di redazione: Jacopo Bassi - Luca Bufarale - Elisa Grandi - Deborah Paci - Fausto Pietrancosta - Matteo Tomasoni - Luca Zuccolo 